\title{
The prognostic significance of large vessel occlusion in stroke patients treated by intravenous thrombolysis
}

\author{
Justyna M. Derbisz ${ }^{1,2 A, B, D, E}$, Marcin Wnuk ${ }^{2 D}$, Tadeusz Popiela ${ }^{2 D}$, Jeremiasz Jagiełła ${ }^{2 D}$, Roman Pułyk ${ }^{2 D}$, \\ Joanna Słowik ${ }^{3 C}$, Tomasz Dziedzic ${ }^{2 D}$, Wojciech Turaj ${ }^{2 D}$, Agnieszka Słowik ${ }^{2 A, C D, E}$ \\ 'Department of Neurology, University Hospital, Krakow, Poland \\ 2Department of Neurology, Jagiellonian University Medical College, Krakow, Poland \\ ${ }^{3}$ Department of Dental Prophylaxis and Experimental Dentistry, Jagiellonian University Medical College, Krakow, Poland
}

\section{Abstract}

Purpose: According to guidelines, to shorten the treatment window, acute ischaemic stroke (AIS) treatment by intravenous thrombolysis (IVT) can be done based on the results of head computed tomography (CT) without contrast. The impact of large vessel occlusion (LVO) on computed tomography angiography (CTA) in stroke prognosis in patients treated IVT or IVT and mechanical thrombectomy (MT), where indicated, has not yet been studied systematically. We investigated the influence of LVO in consecutive AIS patients on haemorrhagic transformation (HT) on CT $24 \mathrm{~h}$ after treatment, $\mathrm{mRS}<2$ on discharge (unfavourable outcome), and in-hospital mortality.

Material and methods: We analysed several parameters within $24 \mathrm{~h}$ after AIS: demographics, risk factors, mRS score pre-stroke, NIHSS upon admission and $24 \mathrm{~h}$ later, several clinical and biochemical parameters, and chronic treatment.

Results: We registered 1209 patients, of whom 362 (29.9\%) received IVT and 108 had MT, where indicated. Admission CTA showed LVO in 197 patients (54.4\%). Multivariate regression analysis showed that the presence of LVO and lower delta NIHSS (NIHSS on admission minus NIHSS 24 hours later) were independent parameters affecting HT risk. Multivariate analysis showed that the presence LVO and also older age, female sex, lower delta NIHSS, HT, stroke-associated infection, CRP levels $\geq 10 \mathrm{mg} / \mathrm{L}$, and higher WBC count affected unfavourable outcome on discharge. LVO did not affect in-hospital mortality.

Conclusions: LVO in AIS patients treated by IVT or IVT and MT affects the risk of HT and unfavourable short-term outcome but not in-hospital mortality.

Key words: acute stroke, thrombolysis, mechanical thrombectomy, outcome.

\section{Introduction}

Intravenous thrombolysis (IVT) by rt-PA or tenecteplase is the only effective pharmacological treatment for acute ischaemic stroke (AIS) [1,2]. Unfortunately, this treatment is efficient only in a small proportion of patients [3]. Time from stroke onset to treatment by IVT is thought to be the most important factor affecting IVT efficacy [3]. Accord- ing to guidelines, to save time, the treatment of AIS by IVT can be done based on the results of head computed tomography (CT) without contrast [1,2]. A few studies, additionally implementing computed tomography angiography (CTA), have shown that large vessel occlusion (LVO) is an important poor prognostic factor in patients with AIS, irrespective of whether they are being treated [4-6] or not treated by IVT [7].

Correspondence address:

Justyna Maria Derbisz, MD, Department of Neurology, University Hospital, 2 Jakubowskiego Str., 30-688 Krakow, Poland, e-mail: justyna.derbisz@gmail.com

Authors' contribution:

A Study design · B Data collection · C Statistical analysis · D Data interpretation · E Manuscript preparation · F Literature search · G Funds collection 
In recent years, mechanical thrombectomy (MT) has become a recommended treatment option for patients with LVO [8-12]. Key studies showing the effectiveness of MT in AIS indicated the advantage of brain vessel neuroimaging before the treatment procedure to show detailed LVO location [8-12]. In our Stroke Unit, we introduced a complex neuroimaging protocol on admission, including computed tomography (CT) with and without contrast and CTA, in all consecutive AIS patients, irrespectively of their treatment perspective and whether or not they declared any history of chronic renal disease. The protocol allowed us to gather immediately data on the presence of LVO, its location, or the presence of vascular malformations. All this information allows easier and safer decision-making about AIS treatment and smoother patient flow during the therapeutic window.

In this study, we analysed the frequency and the influence of LVO on admission CTA in consecutive patients treated by IVT or IVT and MT, where indicated, on shortterm outcome measures (i.e. haemorrhagic transformation [HT] on CT $24 \mathrm{~h}$ after treatment, poor outcome as measured by the mRS 3-6 on discharge, and in-hospital mortality).

\section{Material and methods}

We retrospectively analysed the data prospectively collected in the Krakow Stroke Data Bank (KSDB). The KSDB is a single-centre, hospital-based registry in which clinical, radiological, and genetic data from AIS cases are included. The systematic collection of data was started in 2007. The study design was approved by the Jagiellonian University Ethical Committee (KBET 54/B/2007). The diagnosis of stroke was made according to the definition by Sacco et al. [13]. The collected patient information has been described in detail elsewhere [14]. For the purpose of the present study, we analysed data from participants of KSDB treated with IVT from June 2014 to December 2018. In each included patient we analysed the following data available within $24 \mathrm{~h}$ after stroke: demographics (age and sex); stroke risk factors (hypertension, diabetes mellitus, ischaemic heart disease, atrial fibrillation, hypercholesterolaemia, smoking status, body mass index, and history of stroke); clinical parameters (prestroke independence as measured by the modified Rankin Score [mRS]) [15] (we distinguished patients with $\mathrm{mRS}=0$ [no disability] from those with $\mathrm{mRS}>0$ [at least slight disability]); body temperature on admission (we distinguished patients with body temperature $\geq 37^{\circ} \mathrm{C}$ and with body temperature $<37^{\circ} \mathrm{C}$ ); time from stroke onset to alteplase infusion; and maximum systolic blood pressure and diastolic blood pressures within 24 hours after alteplase infusion. We distinguished patients with maximal systolic blood pressure $>160 \mathrm{~mm} \mathrm{Hg}$ and $\leq 160 \mathrm{~mm} \mathrm{Hg}$ (160 mm Hg was the median value); and patients with maximal diastolic blood pressure $>85 \mathrm{~mm}$ $\mathrm{Hg}$ and $\leq 85 \mathrm{~mm} \mathrm{Hg}$ (85 $\mathrm{mm} \mathrm{Hg}$ was the median value).
Stroke severity was measured on admission and 24 hours later according to the National Institutes of Health Stroke Scale (NIHSS) [16]. Delta NIHSS was defined as the difference between NIHSS score measured upon hospital admission minus the NIHSS score 24 hours after alteplase infusion (lower scores reflect deterioration; higher scores indicate improvement). We also examined post-treatment haemorrhagic complications on CT taken 24 hours poststroke (categorized as patients with or without bleeding) and stroke aetiology according to the Trial of Org10172 in acute Stroke Treatment (TOAST) criteria [17]. We also analysed the presence and location of LVO shown on CTA performed on admission. We analysed LVO location. We also distinguished patients with and without LVO on CTA in large vessels of the brain.

We collected data on the use of antibiotics during hospitalization due to stroke-associated infection (SAI) (we distinguished those with and without SAI). The details about the diagnosis of infections have been reported elsewhere [18].

Biochemical data taken while fasting on the $1^{\text {st }}$ day morning were as follows: CRP levels (increased CRP levels was diagnosed as CRP $\geq 10 \mathrm{mg} / \mathrm{l}$ ); white blood cells (WBC) count; fibrinogen levels; haemoglobin levels; platelet count; glucose levels; creatinine levels, TSH, total cholesterol levels, LDL-cholesterol levels, HDL-cholesterol levels, and triglycerides levels.

We analysed the following early outcome measures: post-treatment haemorrhagic transformation (HT) on computed tomography (CT) taken $24 \mathrm{~h}$ after stroke (we distinguished patients with or without bleeding). The intensity of bleeding was evaluated according to the ECASS II criteria [19]. Unfavourable outcome on discharge was defined as mRS score [15] from 3 to 6 . We also analysed the in-hospital death rate.

\section{Statistical analysis}

Clinical characteristics and outcomes between the groups were compared by the unpaired Student $t$-test or $\chi^{2}$ test, where appropriate. To identify potential independent predictors of outcome, variables with $p$-value $<0.05$ in the univariate regression analysis for the presence of brain haemorrhage, unfavourable outcome as measured by $\mathrm{mRS}$ score from 3 to 6 , or death on discharge were subsequently included into a multivariate regression analysis. All statistical calculations were performed by Statistica software version 13.3 (TIBCO software INC). $P$-values below 0.05 were considered statistically significant.

\section{Results}

During the study period, 1209 patients registered with the KSDB and 362 (29.9\%) received IVT. CTA revealed LVO in 197 patients (54.4\%), and 108 patients had MT performed, where indicated. In detail, 46 patients out of 362 (12.7\%) 


\begin{tabular}{|c|c|c|c|c|c|c|c|c|c|c|c|c|c|c|c|c|c|c|c|c|c|c|c|}
\hline 崩 & & & $\begin{array}{l}\tilde{O} \\
0 \\
0\end{array}$ & & 용. & 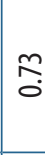 & $\stackrel{\approx}{\sigma}$ & $\bar{\sigma}$ & $\stackrel{\text { ڤ̆ }}{\circ}$ & $\bar{\sigma}$ & $\stackrel{\infty}{\dddot{0}}$ & 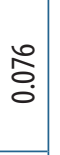 & & ְ. & $\begin{array}{l}\approx \\
\vdots \\
-\end{array}$ & 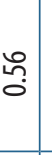 & $\frac{0}{\circ}$ & $\stackrel{ }{\circ}$ & $\begin{array}{l}\bar{\delta} \\
\bar{\delta} \\
\dot{0} \\
\text { v } \\
\end{array}$ & \begin{tabular}{l|l}
$\bar{\delta}$ \\
$\overline{0}$ \\
0 \\
$\overline{0}$ \\
v
\end{tabular} & $\begin{array}{l}\bar{\delta} \\
0 \\
0 \\
\overline{0} \\
v\end{array}$ & 守 & $\begin{array}{l}\bar{\delta} \\
\overline{0} \\
\dot{0} \\
v\end{array}$ \\
\hline 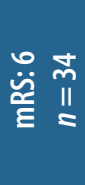 & & ל. & $\begin{array}{l}\underset{尺}{\tilde{\Xi}} \\
\underset{\approx}{\approx}\end{array}$ & & 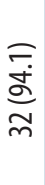 & 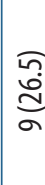 & $\begin{array}{l}\text { न. } \\
\text { d్ } \\
0\end{array}$ & 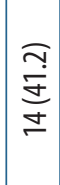 & $\underset{\substack{n \\
\tilde{D}}}{\infty}$ & 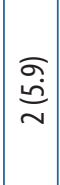 & 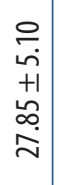 & 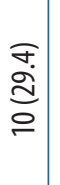 & & 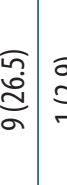 & 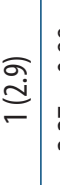 & 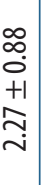 & 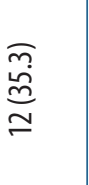 & 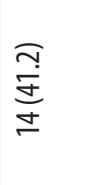 & 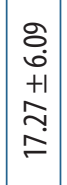 & 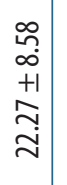 & 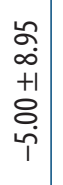 & 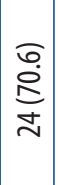 & $\begin{array}{l}\bar{\alpha} \\
\bar{\sigma} \\
\overline{\bar{v}}\end{array}$ \\
\hline 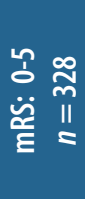 & & 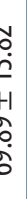 & 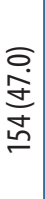 & & 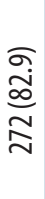 & 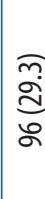 & $\begin{array}{l}\stackrel{\sigma}{d} \\
\underset{N}{\pi}\end{array}$ & $\mid \begin{array}{l}\sigma \\
o \\
\stackrel{d}{\sim} \\
\sigma \\
\sigma\end{array}$ & $\begin{array}{l}\text { శ్ } \\
\tilde{m} \\
\text { g. }\end{array}$ & 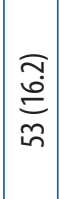 & $\begin{array}{l}\stackrel{m}{+} \\
\dot{+} \\
+1 \\
\infty \\
\stackrel{\sim}{\sim}\end{array}$ & 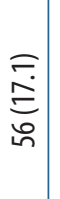 & & 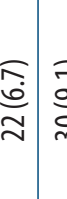 & 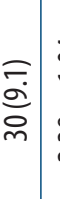 & 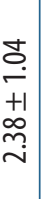 & $\begin{array}{l}\underset{+}{d} \\
\underset{\infty}{d}\end{array}$ & $\begin{array}{l}\underset{\stackrel{\sigma}{d}}{\sigma} \\
\sigma\end{array}$ & 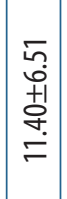 & 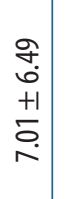 & 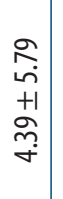 & 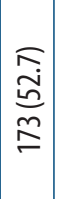 & 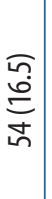 \\
\hline 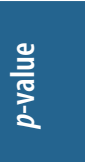 & & & $\begin{array}{l}\stackrel{0}{0} \\
0 \\
0\end{array}$ & & $\begin{array}{l}\bar{\delta} \\
\text { ठ̀ } \\
\dot{v} \\
v\end{array}$ & $\stackrel{m}{0}$ & $\begin{array}{l}\bar{o} \\
\text { o. }\end{array}$ & $\begin{array}{l}0 \\
\bar{\sigma} \\
0 \\
0\end{array}$ & $\bar{\sigma}$ & $\overline{\hat{m}}$ & $\begin{array}{l}\text { oे } \\
0\end{array}$ & $\begin{array}{l}\tilde{\Xi} \\
\tilde{\sigma}\end{array}$ & & $\tilde{\delta}_{\bar{\delta}}^{m}$ & $\widetilde{\widetilde{o}}$ & $\begin{array}{l}: \\
0 \\
0\end{array}$ & 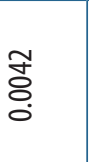 & 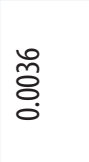 & $\begin{array}{l}\bar{\delta} \\
\bar{\delta} \\
0 \\
\dot{v} \\
v\end{array}$ & $\begin{array}{l}\bar{\delta} \\
\bar{\delta} \\
\bar{v} \\
v\end{array}$ & $\begin{array}{l}\bar{\delta} \\
\bar{\delta} \\
\dot{0} \\
v\end{array}$ & $\begin{array}{l}\bar{\delta} \\
\bar{\delta} \\
0 \\
\dot{v} \\
v\end{array}$ & $\begin{array}{l}\bar{\delta} \\
\text { ठ. } \\
\dot{0} \\
v\end{array}$ \\
\hline 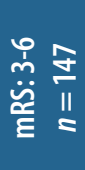 & & 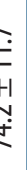 & 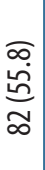 & & 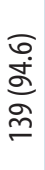 & 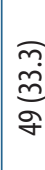 & $\begin{array}{l}\sigma \\
\bar{\Xi} \\
\bar{\sigma} \\
\bar{\sigma}\end{array}$ & $\mid$\begin{tabular}{|l}
$\overline{\bar{\delta}}$ \\
$\tilde{n}$ \\
$\tilde{n}$
\end{tabular} & $\underset{\mathscr{O}}{\stackrel{\Xi}{\sim}}$ & $\begin{array}{c}\sigma \\
\stackrel{\Xi}{\Xi} \\
\stackrel{\sigma}{\sigma}\end{array}$ & $\begin{array}{l}\stackrel{+}{+} \\
+1 \\
\infty \\
\stackrel{\sim}{\sim}\end{array}$ & 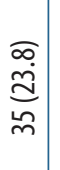 & & 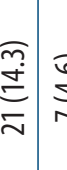 & $\begin{array}{l}\stackrel{\sigma}{+} \\
\stackrel{+}{\sim}\end{array}$ & $\begin{array}{l}\infty \\
0 \\
+1 \\
+1 \\
\end{array}$ & 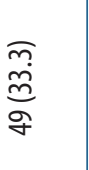 & 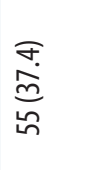 & $\begin{array}{l}\hat{\omega} \\
+1 \\
\tilde{n} \\
\hat{\sigma}\end{array}$ & 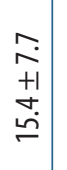 & \begin{tabular}{l}
$\frac{8}{0}$ \\
\multirow{1}{+}{} \\
+1 \\
8 \\
0 \\
0
\end{tabular} & 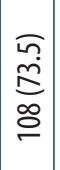 & $\begin{array}{l}\bar{\alpha} \\
\infty \\
\stackrel{0}{n} \\
i n\end{array}$ \\
\hline 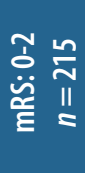 & & $\frac{\dot{y}}{\mathrm{H}}$ & 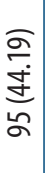 & & 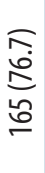 & $\begin{array}{l}0 \\
\text { dై } \\
\text { in }\end{array}$ & 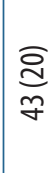 & 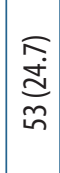 & 定 & 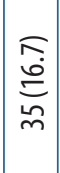 & $\begin{array}{l}\bar{j} \\
+1 \\
+1 \\
\stackrel{\partial}{\nu} \\
\dot{\nu}\end{array}$ & 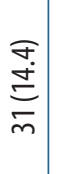 & & 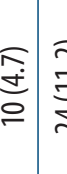 & $\begin{array}{l}\underset{\mathfrak{c}}{\Xi} \\
\stackrel{\Xi}{ \pm}\end{array}$ & 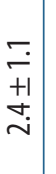 & 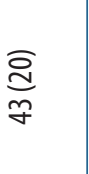 & 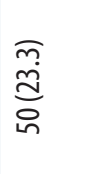 & 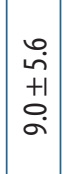 & 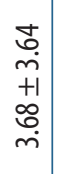 & 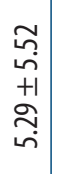 & 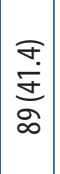 & $\begin{array}{l}\underset{f}{\infty} \\
\infty \\
\infty\end{array}$ \\
\hline 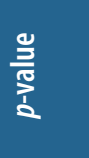 & & : & ণे & & $\stackrel{\circ}{0}$ & $\begin{array}{c}\tilde{\infty} \\
0 \\
0\end{array}$ & : & $\underset{\Xi}{\tilde{\Xi}}$ & 号 & $\bar{\sigma}$ & $\frac{0}{\circ}$ & бু & & $\tilde{a}$ & $\bar{\sigma}$ & $\underset{\mathcal{Z}}{\mathcal{Z}}$ & İ & ộ. & $\begin{array}{l}\bar{\delta} \\
\text { o } \\
\text { d. } \\
\text { v }\end{array}$ & $\begin{array}{l}\bar{\delta} \\
\vdots \\
0 \\
\dot{0} \\
v\end{array}$ & 志 & $\begin{array}{l}\bar{\delta} \\
\bar{\delta} \\
\overline{0} \\
\mathrm{v}\end{array}$ & \\
\hline 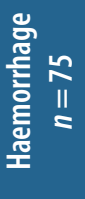 & & $\frac{2}{\dot{2}}$ & 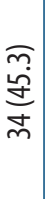 & & $\begin{array}{l}\bar{m} \\
\stackrel{a}{\sigma} \\
\underline{\sigma}\end{array}$ & 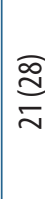 & 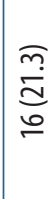 & $\frac{\bar{o}}{\stackrel{p}{0}}$ & 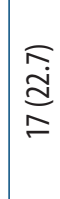 & \begin{tabular}{|c}
$\bar{n}$ \\
$\stackrel{n}{\Xi}$ \\
0 \\
0
\end{tabular} & 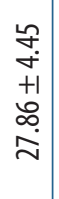 & 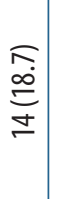 & & $\begin{array}{c}\bar{m} \\
\stackrel{a}{n}\end{array}$ & $\underset{m}{\stackrel{\Xi}{m}}$ & 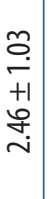 & 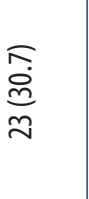 & $\begin{array}{l}\bar{న} \\
\stackrel{n}{\infty} \\
\stackrel{\sim}{\infty}\end{array}$ & 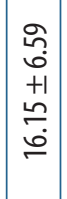 & 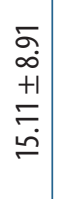 & $\mid \begin{array}{c}\sigma \\
\infty \\
+1 \\
0 \\
\infty \\
0 \\
0\end{array}$ & 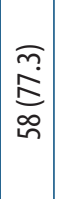 & \\
\hline 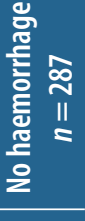 & & 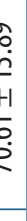 & 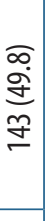 & & 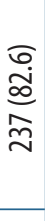 & $\begin{array}{l}\bar{m} \\
\stackrel{2}{2} \\
\bar{\infty}\end{array}$ & $\begin{array}{l}\widetilde{\sigma} \\
\underset{\tilde{D}}{\infty} \\
\infty\end{array}$ & 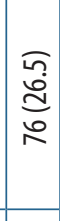 & $\begin{array}{l}\infty \\
\dot{0} \\
\stackrel{0}{0} \\
\overline{0}\end{array}$ & 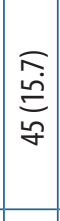 & $\begin{array}{l}0 \\
\dot{+} \\
+1 \\
\dot{\sigma} \\
\stackrel{亠}{\sim}\end{array}$ & 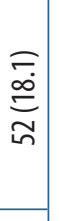 & & 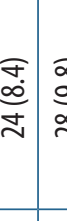 & $\begin{array}{l}\bar{\alpha} \\
\stackrel{0}{0} \\
\stackrel{\sim}{\sim}\end{array}$ & 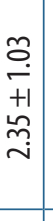 & 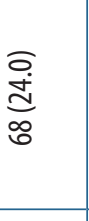 & 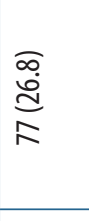 & 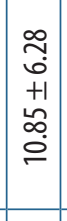 & 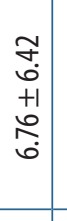 & 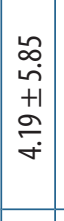 & 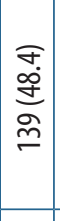 & \\
\hline 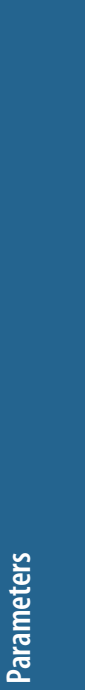 & & 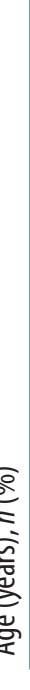 & 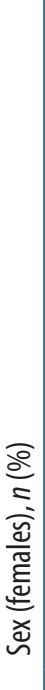 & 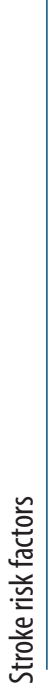 & 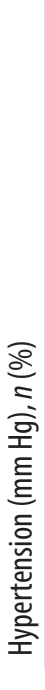 & 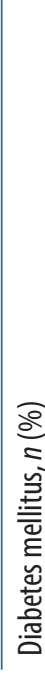 & 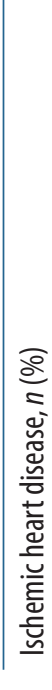 & 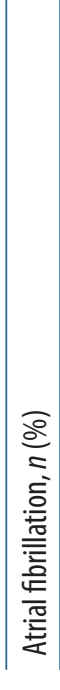 & 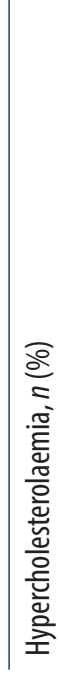 & 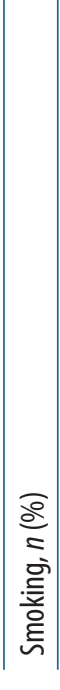 & 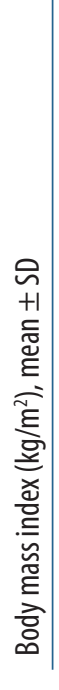 & 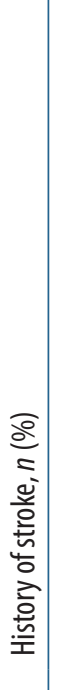 & 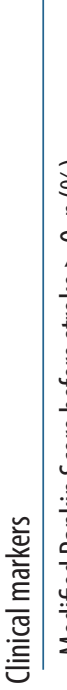 & 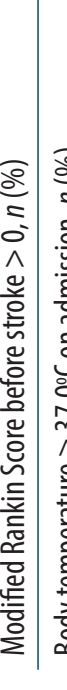 & 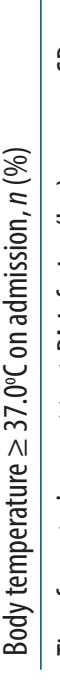 & 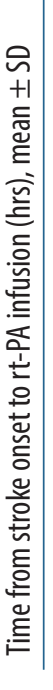 & 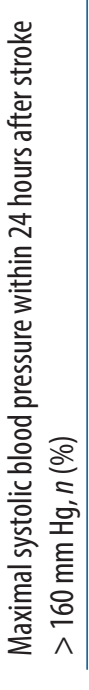 & 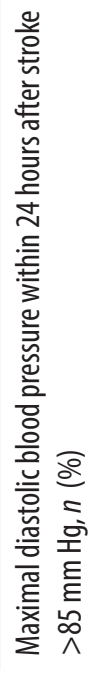 & 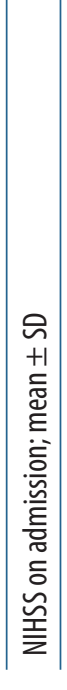 & 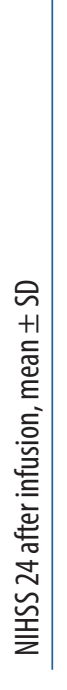 & 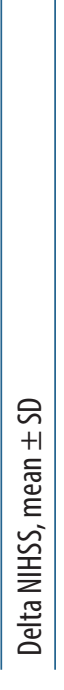 & 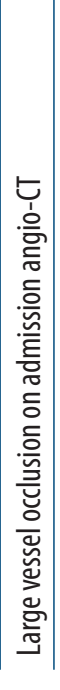 & 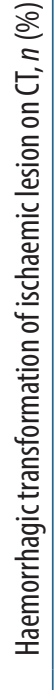 \\
\hline
\end{tabular}




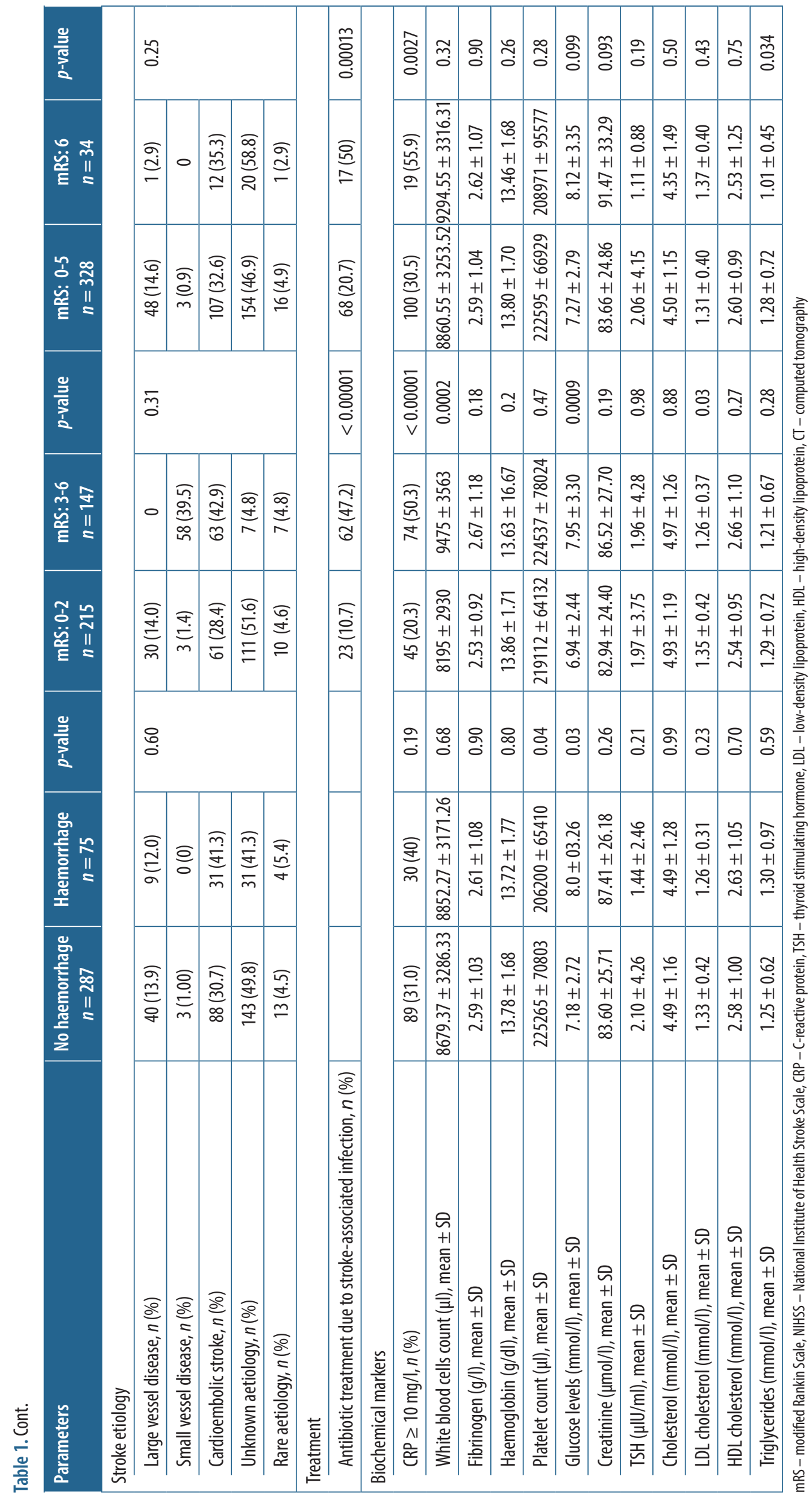


Table 2. Multivariate regression models analysing parameters affecting risk of haemorrhagic transformation on CT (model including or excluding CRP and WBC count), factors affecting unfavourable short-term outcome (mRS: 3-6) and in hospital mortality (mRS =6)

\begin{tabular}{|c|c|c|c|c|c|c|c|}
\hline Parameter & OR & $95 \% \mathrm{Cl}$ & $p$-value & Parameter & OR & $95 \% \mathrm{Cl}$ & $p$-value \\
\hline \multicolumn{4}{|c|}{$\begin{array}{l}\text { Parameters affecting risk of haemorrhage on CT taken } 24 \text { hours after alteplase } \\
\text { infusion }\end{array}$} & \multicolumn{4}{|c|}{$\begin{array}{l}\text { Parameters affecting unfavourable outcome on discharge without CRP, } \\
\text { and WBC count }\end{array}$} \\
\hline \multicolumn{4}{|l|}{ Demographics } & \multicolumn{4}{|l|}{ Demographics } \\
\hline Age (years) & 0.99 & $0.97-1.01$ & 0.29 & Age (years) & 1.05 & $1.02-1.09$ & 0.0013 \\
\hline Sex (females) & 0.87 & $0.48-1.60$ & 0.65 & Sex (females) & 2.44 & $1.21-4.91$ & 0.01 \\
\hline \multicolumn{4}{|l|}{ Stroke risk factors } & \multicolumn{4}{|l|}{ Stroke risk factors } \\
\hline Atrial fibrillation & 1.90 & $0.99-3.62$ & 0.05 & \multirow{2}{*}{ Hypertension (mm Hg) } & 3.01 & $0.93-9.67$ & 0.06 \\
\hline Hypercholesterolemia & 0.67 & $0.35-1.27$ & 0.22 & & \multirow{2}{*}{0.79} & \multirow{2}{*}{$0.37-1.68$} & \multirow{2}{*}{0.54} \\
\hline \multicolumn{4}{|l|}{ Clinical parameters } & Atrial fibrillation & & & \\
\hline \multirow{2}{*}{$\begin{array}{l}\text { Large vessel occlusion on admission } \\
\text { angio-CT }\end{array}$} & \multirow{2}{*}{3.60} & \multirow{2}{*}{$1.93-6.71$} & \multirow{2}{*}{0.000053} & \multirow{2}{*}{$\begin{array}{l}\text { Body mass index }(\mathrm{kg} \\
\text { History of stroke }\end{array}$} & 1.05 & $0.97-1.14$ & 0.22 \\
\hline & & & & & 1.20 & $0.52-2.74$ & 0.67 \\
\hline Delta NIHSS & 0,92 & $0.88-0.96$ & 0.00011 & \multicolumn{4}{|l|}{ Clinical parameters } \\
\hline \multicolumn{4}{|l|}{ Biochemical parameters } & Modified Rankin Score before stroke $>0$ & 2.97 & $0.99-8.89$ & 0.05 \\
\hline Glucose levels (mmol/l) & 1.09 & $0.99-1.19$ & 0.07 & Body temperature $\geq 37.0^{\circ} \mathrm{C}$ on admission & 0.40 & $0.10-1.53$ & 0.18 \\
\hline Platelet count ( $\mu \mathrm{l})$ & 0.70 & $0.46-1.08$ & 0.11 & Maximal systolic blood pressure within & 0.64 & $0.29-1.44$ & 0.28 \\
\hline Parameters affecting unfavourable outcome on & dischar & & & 24 hours after stroke > $160 \mathrm{~mm} \mathrm{Hg}$ & & & \\
\hline Demographics & & & & Maximal diastolic blood pressure within & 1.45 & $0.71-2.98$ & 0.31 \\
\hline Age (years) & 1.06 & $1.02-1.09$ & 0.0011 & 24 hours after stroke $>85 \mathrm{~mm} \mathrm{Hg}$ & & & \\
\hline Sex (females) & 2.47 & $1.17-5.22$ & 0.02 & Delta NIHSS & 0.84 & $0.78-0.90$ & 0.00000030 \\
\hline Stroke risk factors & & & & Large vessel occlusion on admission & 3.68 & $1.28-7.45$ & 0.00027 \\
\hline Hypertension (mm Hg) & 2.80 & $0.83-9.41$ & 0.09 & angio-CT & & & \\
\hline Atrial fibrillation & 0.82 & $0.38-1.80$ & 0.62 & Haemorrhagic transformation on CT & 4.93 & $2.25-10.78$ & 0.000061 \\
\hline Body mass index $\left(\mathrm{kg} / \mathrm{m}^{2}\right)$ & 1.05 & $0.96-1.14$ & 0.28 & 24 hours after admission & & & \\
\hline History of stroke & 1.21 & $0.50-2.94$ & 0.66 & 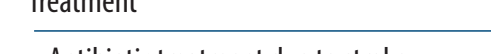 & & & \\
\hline Clinical parameters & & & & $\begin{array}{l}\text { Antibiotic treatment due to stroke- } \\
\text { associated infection, } n(\%)\end{array}$ & 5.88 & $2.79-12.40$ & 0.0000030 \\
\hline Modified Rankin scale score before stroke $>0$ & 3.13 & $0.97-10.12$ & 0.05 & Biochemical parameters & & & \\
\hline Body temperature $\geq 37.0^{\circ} \mathrm{C}$ on admission & 0.29 & $0.07-1.19$ & 0.08 & Glucose levels (mmol/l) & 1.02 & $0.91-1.13$ & 0.75 \\
\hline $\begin{array}{l}\text { Maximal systolic blood pressure within } \\
24 \text { hours after stroke }>160 \mathrm{~mm} \mathrm{Hg}\end{array}$ & 1.54 & $0.72-3.29$ & 0.26 & $\mathrm{LDL}(\mathrm{mmol} / \mathrm{l})$ & 0.69 & $0.30-1.61$ & 0.39 \\
\hline Maximal diastolic blood pressure within & 0.78 & $0.34-1.77$ & 0.55 & Parameters affecting the risk of death at disch & arge & & \\
\hline 24 hours after stroke $>85 \mathrm{~mm} \mathrm{Hg}$ & & & & Demographics & & & \\
\hline Delta NIHSS & 0.87 & $0.78-0.90$ & 0.00000022 & Age (years) & 1.03 & $0.97-1.08$ & 0.34 \\
\hline Large vessel occlusion on admission & 3.12 & $1.48-6.54$ & 0.0026 & Sex (females) & 2.58 & $0.86-7.79$ & 0.09 \\
\hline He morrbaic trancformation & & & & Clinical parameters & & & \\
\hline $\begin{array}{l}\text { Haemorrhagic transformation on CT } \\
24 \text { hours after admission }\end{array}$ & 6.40 & $2.75-14.88$ & 0.000015 & Modified Rankin scale score before & 9.56 & $2.47-37.03$ & 0.0010 \\
\hline Treatment & & & & & & & \\
\hline Antibiotic treatment due to stroke- & 3.83 & $1.73-8.51$ & 0.00093 & Delta NIHSS & 0.78 & $0.71-0.86$ & 0.0000014 \\
\hline associated infection, $n(\%)$ & & & & $\begin{array}{l}\text { Large vessel occlusion on admission } \\
\text { angio-CT }\end{array}$ & 1.57 & $0.55-4.45$ & 0.40 \\
\hline Biochemical parameters & & & & & 676 & $235-1946$ & 000038 \\
\hline CRP $\geq 10(\mathrm{mg} / \mathrm{l})$ & 3.70 & $1.81-7.54$ & 0.00031 & $\begin{array}{l}\text { Haemorrnagic transtormation on cl day } \\
\text { after admission }\end{array}$ & 0.10 & $2.35-19.40$ & 0.00038 \\
\hline White blood cells count $(\mu \mathrm{l})$ & 1.13 & $1.01-1.26$ & 0.04 & Treatment & & & \\
\hline Glucose levels (mmol/l) & 0.99 & $0.88-1.12$ & 0.84 & Antibiotic treatment due to stroke- & 0.96 & $0.32-2.86$ & 0.94 \\
\hline $\mathrm{LDL}(\mathrm{mmol} / \mathrm{l})$ & 0.77 & $0.31-1.93$ & 0.59 & associated infection, $n(\%)$ & & & \\
\hline
\end{tabular}


Table 2. Cont.

\begin{tabular}{|c|c|c|c|}
\hline Parameter & OR & $95 \% \mathrm{Cl}$ & $p$-value \\
\hline \multicolumn{4}{|l|}{ Biochemical markers } \\
\hline CRP $\geq 10(\mathrm{mg} / \mathrm{l})$ & 3.11 & $1.08-8.93$ & 0.03 \\
\hline $\mathrm{TG}(\mathrm{mmol} / \mathrm{l})$ & 0.30 & $0.08-1.12$ & 0.07 \\
\hline \multicolumn{4}{|c|}{ Parameters affecting risk of death at discharge, without CRP } \\
\hline \multicolumn{4}{|l|}{ Demographics } \\
\hline Age (years) & 1.03 & $0.98-1.09$ & 0.21 \\
\hline Sex (males) & 2.40 & $0.81-7.06$ & 0.11 \\
\hline \multicolumn{4}{|l|}{ Clinical parameters } \\
\hline $\begin{array}{l}\text { modified Rankin scale score before } \\
\text { stroke }>0\end{array}$ & 8.75 & $2.36-32.44$ & 0.0011 \\
\hline Delta NIHSS & 0.79 & $0.72-0.87$ & 0.0000020 \\
\hline $\begin{array}{l}\text { Large vessel occlusion on admission } \\
\text { angio-CT }\end{array}$ & 1.75 & $0.63-4.86$ & 0.28 \\
\hline $\begin{array}{l}\text { Haemorrhagic transformation on CT day } \\
\text { after admission }\end{array}$ & 6.65 & $2.36-18.75$ & 0.00032 \\
\hline \multicolumn{4}{|l|}{ Treatment } \\
\hline $\begin{array}{l}\text { Antibiotic treatment due to stroke- } \\
\text { associated infection, } n(\%)\end{array}$ & 1.38 & $0.50-3.81$ & 0.53 \\
\hline \multicolumn{4}{|l|}{ Biochemical parameters } \\
\hline $\mathrm{TG}(\mathrm{mmol} / \mathrm{l})$ & 0.35 & $0.11-1.17$ & 0.09 \\
\hline
\end{tabular}

NIHSS - National Institute of Health Stroke Scale, CRP - C-reactive protein, TG - triglycerides, LDL - lowdensity lipoprotein, CT - computed tomography

had LVO in the symptomatic carotid artery. Among these, 18 (39.1\%) had IVT, and 28 (60.9\%) had IVT and subsequent MT. In 91 patients (25.1\%), LVO was in the M1 region of the symptomatic middle cerebral artery (MCA). Among these, 36 (39.7\%) had IVT, and 55 (60.3\%) had IVT and MT. In 36 patients (9.9\%), LVO was in the M2 region of symptomatic MCA. Among these, 21 (58.4\%) had IVT, and $15(41.6 \%)$ had IVT and subsequent MT. In 8 patients (2.2\%), LVO was in the M3 region of symptomatic MCA. Among these, 6 (75\%) had IVT, and 2 (25\%) had IVT and MT. In 16 patients (4.4\%), LVO was in the symptomatic basilar artery. Among these, 8 cases had IVT and 8 had IVT plus MT. In 128 patients (35.6\%), CTA did not show LVO, and in another 37 patients (10.2\%) CTA showed arterial atherosclerotic changes in brain arteries without AC.

Haemorrhagic transformation on CT taken $24 \mathrm{~h}$ after stroke was found in 75 patients. According to the ECASS II classification, 25 patients (6.9\%) developed small petechiae within the infarcted area, 24 patients $(6.6 \%)$ had more confluent petechiae within the infarcted area, 14 (3.9\%) had haemorrhage not exceeding $30 \%$ of the infarcted area with some mild space-occupying effect, and 12 (3.3\%) had brain haemorrhage exceeding $30 \%$ of the infarct volume with significant space-occupying effect. Groups of patients with and without HT did not differ with respect to age and sex distribution. Univariate analysis showed that LVO on admission CTA, AF, hypercholesterolaemia, lower delta
NIHSS, higher platelet count, and higher glucose levels correlated with HT (Table 1). Multivariate analysis showed that LVO on admission CTA and lower delta NIHSS were independent factors that affected HT risk (Table 2).

In total 147 patients (40.5\%) had an unfavourable outcome, as measured by mRS: 3-6 on discharge. Univariate analysis showed that these patients were significantly older and more often female, more often had LVO on admission CTA, had higher incidence rates of hypertension and AF, had higher body mass index $\left(\mathrm{kg} / \mathrm{m}^{2}\right)$, were more likely to have a history of stroke, more often had body temperature $\geq 37^{\circ} \mathrm{C}$ on admission, and more often maximal systolic blood pressure $>160 \mathrm{~mm} \mathrm{Hg}$ and maximal diastolic blood pressure $>85 \mathrm{~mm} \mathrm{Hg}$ within $24 \mathrm{~h}$ after stroke. Patients with unfavourable outcomes were more likely to have mRS scores above 0 pre-stroke or HT on CT, had lower NIHSS than those with a favourable outcome, were more likely to require treatment with antibiotics due to SAI, and were more likely to have CRP levels $\geq 10 \mathrm{mg} / \mathrm{l}$, higher WBC counts, and higher glucose and lower LDL-cholesterol levels (Table 1).

Multivariate analysis showed that the presence LVO on admission CTA, older age, female sex, lower delta NIHSS, HT on CT taken 24 h post-stroke, antibiotic treatment due to SAI, more likely CRP levels $\geq 10 \mathrm{mg} / \mathrm{l}$, and higher WBC count affected unfavourable outcome on discharge (Table 2).

Multivariate analysis, excluding CRP levels and WBC count (inflammatory markers), showed a similar profile of factors affecting unfavourable outcome on discharge (i.e. LVO, older age, female sex, lower delta NIHSS, HT on CT taken $24 \mathrm{~h}$ post-stroke, antibiotic treatment due to SAI) (Table 2).

Thirty-four (9.4\%) of the patients died before discharge. Patients who died were older and more likely to be female. Univariate analysis showed that they more often had LVO on admission CTA. Compared to their surviving counterparts, patients who died were more likely to score above 0 on the mRS before the stroke, had lower delta NIHSS, and presented more frequently with HT on CT taken with $24 \mathrm{~h}$ post-treatment. They were also significantly more likely to use antibiotic treatment due to SAI. Concerning the biochemical parameters, they presented with CRP levels $\geq 10 \mathrm{mg} / \mathrm{l}$ more often and had lower triglyceride levels (Table 1).

Multivariate analysis showed that an mRS above 0 before admission, lower delta NIHSS, HT on CT taken $24 \mathrm{~h}$ after IVT, and CRP levels $\geq 10 \mathrm{mg} / \mathrm{l}$ were independent factors that affected the risk of death up to discharge. Multivariate analysis after the exclusion of CRP did not change the profile of independent factors affecting in-hospital mortality (Table 2).

\section{Discussion}

We found that in patients with AIS treated by IVT solely or IVT and MT, where indicated according to current recommendations $[1,2]$, the presence of LVO on admission CTA 
was an independent factor affecting the risk of HT on CT taken $24 \mathrm{~h}$ after stroke and unfavourable outcome on discharge as measured by the mRS: 3-6, but not in-hospital mortality.

A few studies performed in patients treated by IVT solely $[4,5]$ or IVT and intraarterial thrombolysis as bridging therapy [6], before the era of MT, indicated that the LVO on CTA affected poor prognosis. The prognostic significance of the presence of LVO on admission CTA in consecutive patients treated by IVT or IVT and MT, where indicated, was not studied systematically.

According to guidelines, to shorten the time window, the treatment of AIS by IVT can be done based on the results of the head CT without contrast. Our results strengthen the need to perform additionally admission CTA of the brain arteries to show the presence and exact location of LVO in the brain artery. This knowledge is not only helpful in preparing individual treatment plans but also, based on the results of the present study, in predicting short-term prognosis. We would like to emphasize that in our Stroke Unit, in an emergency setting, to shorten the therapeutic window maximally and based on current guidelines $[1,2]$, we do not measure creatinine levels routinely on admission. Only patients who report chronic renal failure on admission have their neuroimaging procedures scheduled individually, after evaluation of the blood parameters of renal efficiency and a consultation with a nephrologist. In all other patients, creatinine levels are routinely studied on the next morning after the admission. In the presented group of patients, we recorded a mean creatinine level of $84.4 \pm 25.8 \mu \mathrm{mol} / 1$ (median value, $81 \mu \mathrm{mol} / \mathrm{l}$; IQR, $68-97 \mu \mathrm{mol} / \mathrm{l}$; min-max values, 27 and $218 \mu \mathrm{mol} / \mathrm{l})$. In patients with creatinine levels outside the normal range, consultation with a nephrologist was routinely scheduled, and treatment was introduced. Nobody from the presented group required dialysis.

Haemorrhagic transformation on CT in AIS patients treated by IVT as an early outcome measure was analysed previously by several authors [20-33]. Most of these were analysed as symptomatic haemorrhage according to ECASS II criteria (i.e. the presence of any haemorrhage on CT not detected on previous CT, combined with neurological deterioration of 4 points or more in NIHSS from baseline) [19]. The prevalence of symptomatic haemorrhages in the studies varied from $3.9 \%$ [27] to $8.4 \%$ [23]. In our study, we were able to find only 12 symptomatic haemorrhages according to the above-mentioned criteria (3.3\%), and we were not able to find any significant differences between patients with and without symptomatic haemorrhage (data not shown). When we compared patients with and without HT on CT taken $24 \mathrm{~h}$ after stroke, despite taking into account any concomitant changes in neurological deficit, in a multivariate regression analysis, we found that LVO and deterioration of the neurological deficit after treatment affected its risk. Interestingly, our results are in line with previous studies published on this topic [20,29-31], showing the correlation of symptomatic brain haemorrhage with neurological deficit.

In our study, we also measured 2 different early outcome measures: unfavourable outcome (defined as the mRS from 3 to 6 on discharge) and in-hospital mortality. Interestingly, many different early outcome measures were studied so far in AIS patients treated by IVT, such as the following: early recanalization [22,34], in-hospital mortality $[30,35]$, in-hospital mortality or discharge to a nursing home [29], neurological deterioration as measured by an increase of NIHSS by at least 4 points $24 \mathrm{~h}$ after stroke $[24,29,36]$, at least by 8 points on day 7 after stroke $[24,29]$, or deterioration by at least 2 points between day 0 and 5 [21], and mRS on discharge [37]. In those studies, most of the authors analysed the influence of a single chosen parameter on the studied early outcome measure. For example, Kimura et al. showed that AF is the only factor influencing the lack of recanalization, and the lack of recanalization correlated with neurological worsening [34]. Sung et al. showed that mortality on discharge in severe stroke was similar in those with and without AF [30]. Jovanowic et al. showed that early outcome measures are similar in males and females [24], while Al Husain confirmed the lack of correlation between sex and recanalization rate [22]. Tang confirmed that diabetes mellitus affects unfavourable outcomes $24 \mathrm{~h}$ after stroke, and on day 7 after stroke [29]. Only 2 authors were able to build up logistic regression models showing independent factors affecting in-hospital mortality (age $\geq 80$ years, aphasia, conscious disturbances, hypertension, the presence of haemorrhage on CT, and pneumonia) $[20,29]$ and inhospital mortality or discharge to a nursing facility (older age, longer hospital stay, and large cerebral infarct) [38].

In our study, we showed that unfavourable outcome as measured by mRS: 3-6 on discharge, but not in-hospital, is affected by the presence of LVO on admission CTA; lower delta NIHSS and HT on CT taken $24 \mathrm{~h}$ after stroke are common disadvantageous factors affecting both outcome measures in the present study. Unfavourable outcome was also related with antibiotic treatment due SAI, irrespectively of including or excluding markers of inflammation into logistic regression models. Interestingly, as was shown previously [39], age and sex affected the unfavourable outcome but not in-hospital mortality.

Our study is mainly limited by its retrospective analysis. The risk of selection bias is possible because we included only patients who agreed to participate in the study. We also analysed outcome measures on discharge, and, unfortunately, we did not collect detailed information about the length of hospital stay; in Poland, patients with AIS are hospitalized for at least 9 days or until death. Another limitation is the relatively long recruitment period, lasting 5 years, so this long period might increase the number of confounding factors because the approach to stroke treatment changes rapidly over time. 


\section{Conclusions}

In AIS patients treated by IVT solely or by IVT and MT, where indicated according to current guidelines, LVO of the brain vessels shown on admission CTA affects the risk of HT or unfavourable outcome on discharge, but not inhospital mortality.

\section{Conflict of interest}

The authors report no conflict of interest.

\section{References}

1. Powers WJ, Rabinstein AA, Ackerson T, et al. 2018 guidelines for the early management of patients with acute ischemic stroke: a guideline for healthcare professionals from the American Heart Association/American Stroke Association. Stroke 2018; 49: e46-e99.

2. Correction to: Guidelines for the early management of patients with acute ischemic stroke: 2019 update to the 2018 guidelines for the early management of acute ischemic stroke: A guideline for healthcare professionals from the American Heart Association/American Stroke Association. Stroke 2019; 50: e440-e441.

3. Less K, Bluhmki E, Kummer R, et al. Time to treatment with intravenous alteplase and outcome in stroke: an updated pooled analysis of ECASS, ATLANTIS, NINDS, and EPITHET trials. Meta-analysis. Lancet 2010; 375: 1695-1703.

4. Puetz V, Dzialdowski I, Hill MD, et al. Malignant profile detected by CT sangiographic information predicts poor prognosis despite thrombolysis within three hours from symptom onset. Cerebrovasc Dis 2010; 29: 584-591.

5. Rai A, Cline B, Williams E, et al. Intravenous thrombolysis outcomes in patients presenting with large vessel acute ischemic strokes - CT angiography based prognosis. J Neuroimaging 2015; 25: 239-242.

6. Zhu W, Xiao L, Lin M, et al. Large-vessel occlusion is associated with poor outcome in stroke patients aged 80 years or older who underwent intravenous thrombolysis. J Stroke Cerebrovasc Dis 2016; 25: 2712-2716.

7. Smith WS, Lev MH, English JD, et al. Significance of large vessel intracranial occlusion causing acute ischemic stroke and TIA. Stroke 2009; 40: 3834-3840

8. Berkhemer OA, Fransen PS, Beumer D, et al. A randomized trial of intraarterial treatment for acute ischemic stroke. N Engl J Med 2015; 372: 11-20

9. Jovin T G, Chamorro A, Cobo E, et al. Thrombectomy within 8 hours after symptom onset in ischemic stroke. N Engl J Med 2015; 372: 22962306

10. Campbell BCV, Mitchell PJ, Kleinig TJ, et al. Endovascular therapy for ischemic stroke with perfusion-imaging selection. N Engl J Med 2015; 372: 1009-1018.

11. Saver JL, Goyal M, Bonafe A, et al. Stent- retriever thrombectomy after intravenous t-PA vs. t-PA alone in stroke. N Engl J Med 2015; 372: $2285-2295$

12. Goyal M, Demchuk AM, Menon BK, et al. Randomized assessment of rapid endovascular treatment of ischemic stroke. N Engl J Med 2015; 372: 1019-1030.

13. Sacco RL, Kasner SE, Broderick JP, et al. American Heart Association Stroke Council, Council on Cardiovascular Surgery and Anesthesia;
Council on Cardiovascular Radiology and Intervention; Council on Cardiovascular and Stroke Nursing; Council on Epidemiology and Prevention; Council on Peripheral Vascular Disease; Council on Nutrition, Physical Activity and Metabolism. An updated definition of stroke for the 21st century: a statement for healthcare professionals from the American Heart Association/American Stroke Association. Stroke 2013; 44: 2064-2089.

14. Derbisz J, Brzegowy P, Pulyk R, et al. Long-term outcome of acute ischemic stroke with unruptured intracranial aneurysm treated with intravenous thrombolysis. J Vasc Med Surg 2020; 8: 399.

15. Van Swieten J, Koudstaal P, Visser M, et al. Interobserver agreement for the assessment of handicap in stroke patients. Stroke 1988; 19: 604-607.

16. Brott T, Adams HP, Olinger CP, et al. Measurements of acute cerebral infarction: a clinical examination scale. Stroke 1989; 20: 864-870.

17. Adams HP Jr, Bendixen BH, Kappelle LJ, et al. Classification of subtype of acute ischemic stroke: Definition for use in a multicenter clinical trial. Stroke 1993; 24: 35-41.

18. Nowak K, Derbisz J, Peksa J, et al. Post-stroke infection in acute ischemic stroke patients treated by mechanical thrombectomy does not affect long-term outcome. Adv Intern Cardiol 2020; 16: 452-459.

19. Hacke W, Kaste M, Fieschi C, et al. Randomized double-blind placebo-controlled trial of thrombolytic therapy with intravenous alteplase in acute ischemic stroke (ECAS II). Second European-Australasian Acute Stroke Study Investigators. Lancet 1998; 352: 1245-1251.

20. Al-Khaled M, Matthis C, Eggers J. Predictors of in-hospital mortality and the risk of symptomatic intracerebral hemorrhage after thrombolytic therapy with recombinant tissue plasminogen activator in acute ischemic stroke. J Stroke Cerebrovasc Dis 2014; 23: 7-11.

21. Kim DH, Lee DS, Nah HW, et al. Clinical and radiological factors associated with unfavorable outcome after intravenous thrombolysis in patients with mild ischemic stroke. BMC Neurol 2018; 18: 30.

22. Al-Hussain F, Hussain MS, Molina C, et al. Does the sex of acute stroke patients influence the effectiveness of rt-Pa? BMC Neurol 2014; $14: 60$.

23. Meseguer E, Mazighi M, Labreuche J, et al. Outcomes of intravenous recombinant tissue plasminogen activator therapy according to gender. Stroke 2009; 40: 2104-2110.

24. Jovanović DR, Beslać-Bumbasirević LJ, Budimkić M, et al. Do women benefit more from systemic thrombolysis in acute ischemic stroke? A Serbian experience with thrombolysis in ischemic stroke (SETIS) study. Clin Neurol Neurosurg 2009; 111: 729-732.

25. Maestrini I, Strbian D, Gautier S, et al. Higher neutrophil counts before thrombolysis for cerebral ischemia predict worse outcomes. Neurology 2015; 85: 1408-1416. 
26. Liu M, Pan Y, Zhou L, et al. Predictors of post- thrombolysis symptomatic intracranial hemorrhage in Chinese patients with acute ischemic stroke. PLoS One 2017; 12: e0184646.

27. Liu SY, Cao WF, Wu LF, et al. Effect of glycated hemoglobin index and mean arterial pressure on acute ischemic stroke prognosis after intravenous thrombolysis with recombinant tissuse plasminogen activator. Medicine 2018; 97: e13216.

28. Nardi K, Engelter S, Strbian D, et al. Lipid profiles and outcome in patients treated by intravenous thrombolysis for cerebral ischemia. Neurology 2012; 79: 1101-1108.

29. Tang H, Zhang S, Yan S, et al. Unfavorable neurological outcome in diabetic patients with acute ischemic stroke is associated with incomplete recanalization after intravenous thrombolysis. J Neurointerv Surg 2016; 8: 342-346.

30. Sung SF, Chen YW, Tseng MC, et al. Atrial fibrillation predicts good functional outcome following intravenous tissue plasminogen activator in patients with severe stroke. Clin Neurol Neurosurg 2013; 115: 892-895.

31. Seet R, Zhang Yi, Wijdicks E F, et al. Relationship between chronic atrial fibrillation and worse outcomes in stroke patients after intravenous thrombolysis. Arch Neurol 2011; 68: 1454-1458.

32. Padjen V, Bodenant M, Jovanovic DR, et al. Outcome of patients with atrial fibrillation after intravenous thrombolysis for cerebral ischaemia. J Neurol 2013; 260: 3049-3054.

33. Awadh M, MacDougall N, Santosh C, et al. Early recurrent ischemic stroke complicating intravenous thrombolysis for stroke: incidence and association with atrial fibrillation. Stroke 2010; 41: 1990-1995.

34. Kimura K, Iguchi Y, Yamashita S, et al. Atrial fibrillation as an independent predictor for no early recanalization after IV-t-PA in acute ischemic stroke. J Neurol Sci 2008; 267: 57-61.

35. Tseng YJ, Hu RF, Lee ST, et al. Risk factors associated with outcomes of recombinnt tissue plasminogen activator therapy in patients with acute ischemic stroke. Int J Environ Res Public Health 2020; 17: 618.

36. Foell RB, Silver B, Merino JG, et al. Effects of thrombolysis for acute stroke in patients with pre-existing disability. CMAJ 2003; 169: 193-197.

37. Förster A, Gass A, Kern R, et al. Gender differences in acute ischemic stroke: etiology, stroke patterns and response to thrombolysis. Stroke 2009; 40: 2428-2432.

38. Tian MJ, Tayal AH, Schlenk EA. Predictors of poor hospital discharge outcome in acute stroke due to atrial fibrillation. J Neurosci Nurs 2015; 47: 20-26.

39. Wiszniewska M, Fryze W, Wiśniewska A, et al. Sex-related differences among ischaemic stroke patients treated with intravenous thrombolysis in Poland. Neurol Neurochir Pol 2020; 54: 272-276. 